\title{
Analysis of cooling and heating system with Peltier cell
}

\author{
Wieslaw Lyskawinski ${ }^{1, *}$, Wojciech Szelag $^{1}$ \\ ${ }^{1}$ Poznan University of Technology, Institute of Electrical Engineering and Electronics, Piotrowo 3A 60-965 Poznan, Poland
}

\begin{abstract}
The article presents a developed cooling/heating system with a Peltier cell. This system is intended for local improvement of thermal comfort. The interaction between the Peltier module, a water block and the heat sink with a fan has been investigated. A thermal model of the cooling/heating system has been developed, as well as software for determining parameters of the Peltier modules and for analysis of the system operation conditions has been implemented. The obtained results of calculations were compared with the results of experimental tests.
\end{abstract}

\section{Introduction}

Peltier cells are thermoelectric elements in which, thanks to the use of the Peltier phenomenon, it is possible to absorb thermal energy through one side of the module and to extract it through the other side $[1,2]$. The Peltier modules are used in households [3, 4], medicine, army, in space research, heat pumps, in air dryers or in cooling systems [5]. An interesting application of the Peltier modules, which uses both heating and cooling capacities of the cells, is thermally regulated clothing [6].

The article proposes the use of the cooling and heating capacities of the module for producing a small, temperature-controlled zone in its environment. The proposed solution may be an alternative to using large and expensive air conditioning systems in buildings.

In order to test the cooling and heating capacities of systems with Peltier modules, an algorithm and software for determination of the cell parameters, as well as for analysis and design of cooling/heating systems, have been developed. The algorithm and software have been verified by carrying out tests of the designed system on the built-in measuring stand.

\section{Cooling/heating system}

The developed prototype of a cooling and heating system is shown in Fig. 1. It provides for the possibility of cooling the hot side of the cell using a water block or a heat sink. In order to increase the thermal reaction speed of the system, low heat capacity heat sinks used in computer equipment for cooling the processors were applied. Two auxiliary copper blocks were placed on the built-in test stand to measure the thermal power consumed by and extracted from the Peltier cell. Temperature sensors PT 100 and the LabVIEW measuring system were used for temperature measurement.

\section{System analysis algorithm}

On the basis of the circuit model of thermal phenomena and Peltier module parameters, the cooling power $P_{c}$ and heating power $P_{h}$ of the cell can be determined [7]:

$$
P_{i}=2 k\left[\alpha I T_{1}+0.5 b I^{2} \rho / \sigma-\lambda\left(T_{2}-T_{1}\right) \sigma\right]
$$

where: $b=-1$ for $P_{i}=P_{c}, b=1$ for $P_{i}=P_{h}, I$ is the current, $T_{2}, T_{1}$ represent the temperature of the hot and cold surface of the cell, respectively, $\sigma$ is the cell geometric factor, $k$ is the number of pairs of semiconductor columns in the cell, $\alpha$ is the Seebeck coefficient, $\lambda$ is the thermal conductivity, $\rho$ is the resistivity. The values of the last three parameters depend on temperature.

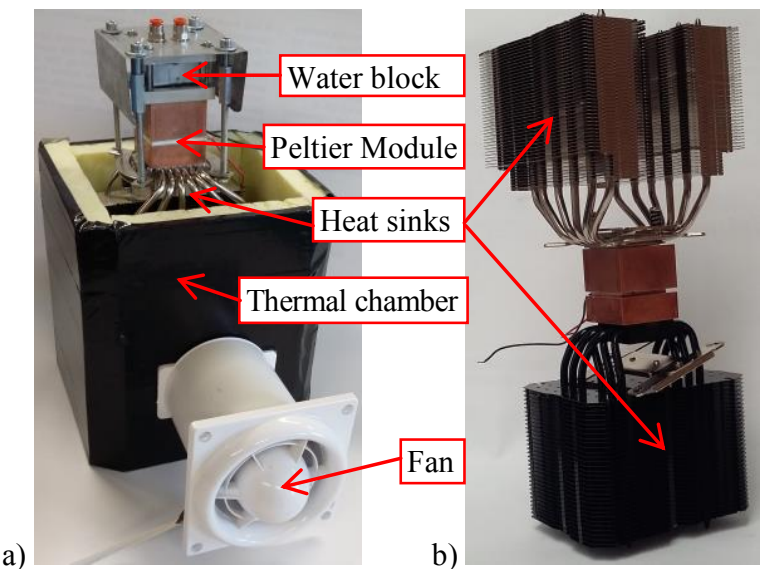

Fig. 1. Cooling and heating system with: a) heat sink and water block, b) two heat sinks.

To determine the temperature of both sides of the cell and selected components of the system, a circuit model of thermal phenomena has been used. The developed equivalent thermal circuit of the systems from Fig. 1 is shown in Fig. 2, where $P_{c}, P_{h}$ represent the thermal power of the cold and hot cell sides, respectively; $R_{t h P}$, $R_{t h C u}, R_{t h R}, R_{t h W B}, R_{t h P a r}$ are the thermal resistances of the thermal paste, copper block, heat sink, water block, thermal insulation of the sidewalls of the copper block, 
respectively; $T_{1}, T_{2}, T_{A}, T_{c}$ show the temperature of the Peltier cell surfaces, the temperature of the ambience and of the cold air at the output of the system, respectively. The method of calculating the equivalent circuit parameters is discussed in detail in [7].

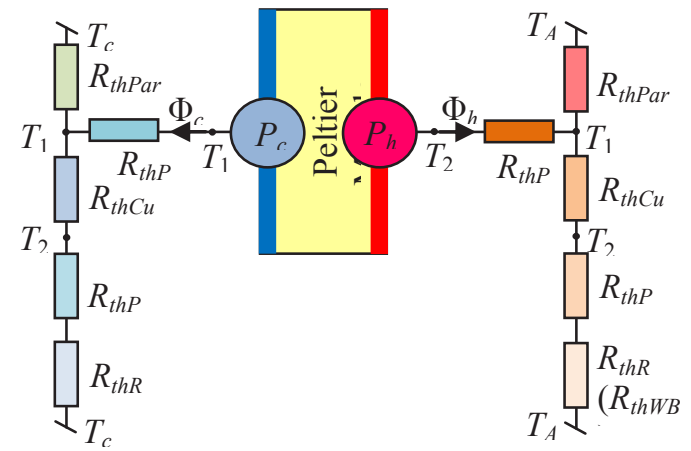

Fig. 2.Thermal equivalent circuit of cooling/heating system.

On the basis of the presented model of thermal phenomena, the software in the $\mathrm{C \#}$ programming language for analysing a cooling/heating system has been developed. It allows the determination of parameters of the equivalent circuit diagram, the thermal input and output power of the Peltier module, as well as the temperature distribution and efficiency of the system. The view of the computer program interface is shown in Fig. 3.

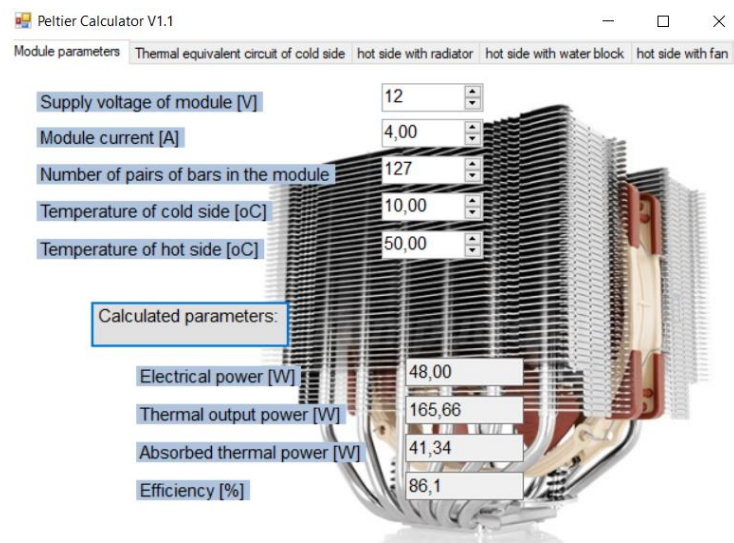

Fig. 3. Main form of the computer program.

\section{Simulation and laboratory tests}

The simulation and experimental tests of the cooling/ heating system with Peltier modules of such types as TEC127085, TES1-24106, TEC1-12726 have been carried out. Due to the limited volume of the article, only selected research results have been presented. Fig. 4 shows the comparison of the calculated and measured temperature dependences $T_{1}(I)$ and $T_{2}(I)$ on the cold and hot areas of the cell surface. During the tests, in the process of being cooled, the radiator was placed in the closed thermal chamber, and the hot side of the cell was cooled with a water block (Fig. 1a). Fig. 5 shows the cooling capacity of the same system, but with the air inlet and outlet of the thermal chamber being open. The influence of $v_{p}$ air velocity on the temperature at the output of the system was investigated. The air speed at the output of the system was adjusted by changing the rotational speed of the fan. The measurements were repeated for two ways of cooling the hot side of the cell and the selected module types.

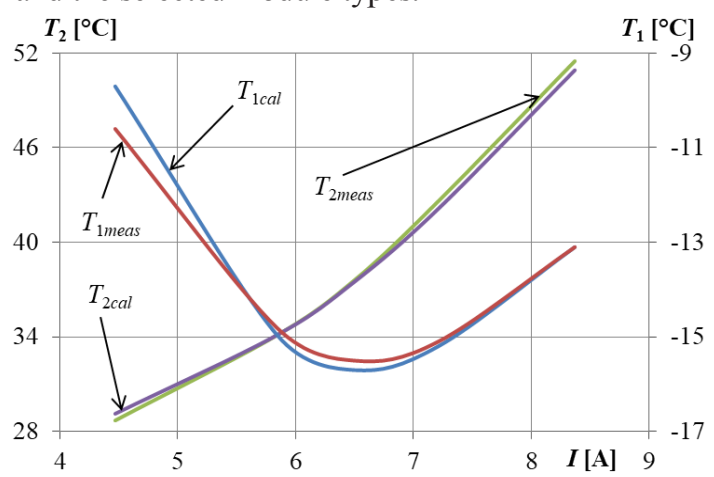

Fig. 4. Comparison of the calculated and measured temperatures $T_{1}$ and $T_{2}$ on the cold and hot sides of a TEC-127085 type cell.

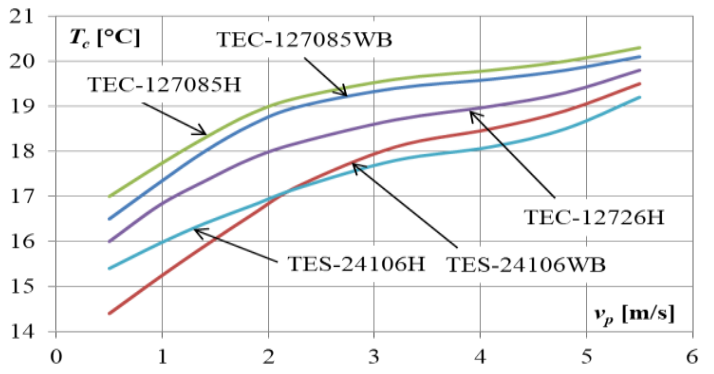

Fig. 5. Comparison of cooling capacity of exhaust air for the systems with a water block (WB) or a heat sink $(\mathrm{H})$.

\section{Conclusions}

Good compatibility between the results of the calculations and experimental research has been obtained. Therefore, the usefulness of the developed algorithm and software for the analysis of thermal phenomena and the design of cooling/heating systems with the Peltier cell has been demonstrated. The performed tests confirm also the usefulness of the Peltier cells in microsystems to improve thermal comfort (local reduction/increase of temperature). The air temperature at the output of the system is regulated by changing the current of the Peltier cell and the rotational speed of the fan. The proposed system is cheap, quiet and ecological (no compressor and environmentally harmful coolant). For this reason, it can be an alternative to large and expensive classic air conditioning systems.

\section{References}

1. J. Goldsmid, Introd. to Thermoel., Springer, (2009)

2. J. Patel, M. Patel, J. Patel, H. Modi, Int. J. Sc. \& Tech. Res. 5, 73 (2016)

3. S. Manikadan, S.C. Kaushik, Elsevier 67, 52 (2015)

4. D.M. Rowe, Thermoelectrics Handbook - Macro to Nano (Taylor and Francis, CRC Press 2006)

5. M. Jaworski, M. Bednarczyk, M. Czachor, Appl. Therm. Eng. 96, 527 (2016)

6. K. K. Vistakula, Patent US8397518 B1 (2012)

7. W. Lyskawinski, W. Szelag, PUT Acad. J., Elec. Eng. 95, 231 (2018) 